\title{
Angiotensinogen Excretion in Rat Urine: Effects of Lipopolysaccharide Treatment and Sodium Balance
}

\author{
Masashi Yoshiya, Yasuhiro Tsutsui ${ }^{\ddagger}$, Norio Itoh and Hiroshi Okamoto* \\ Department of Pharmacology, Faculty of Pharmaceutical Sciences, Kobe-Gakuin University, \\ Ikawadani-cho, Nishi-ku, Kobe 65l-21, Japan \\ Received April 3, 1991 Accepted May 29, 1991
}

\begin{abstract}
Rat urine was found to contain a component showing cross-reactivity with antibody against rat plasma angiotensinogen. Sodium dodecyl sulfate polyacrylamide gel electrophoresis of rat urine revealed antigenic bands corresponding to the molecular weights of plasma angiotensinogen. The urinary angiotensinogen excretion in $8 \mathrm{rats}$, determined by direct radioimmunoassay, was $2.70 \pm 0.21 \mu \mathrm{g} / \mathrm{day}$. Induction of acute inflammation in rats by injection of lipopolysaccharide caused about a 7 -fold increase of urinary angiotensinogen excretion in the $24 \mathrm{hr}$ after injection, with a concomitant elevation of plasma angiotensinogen. Neither sodium depletion nor loading by a low- or high-sodium diet altered the urinary excretion of angiotensinogen. These results suggest that the angiotensinogen present in rat urine is derived from that in plasma, although the level of excretion is too low to have any influence on the plasma level of angiotensinogen.
\end{abstract}

Angiotensinogen, a plasma protein synthesized mainly by the liver, is cleaved by renin to release a decapeptide, angiotensin I. Angiotensin $\mathrm{I}$ is further hydrolyzed by a convertingenzyme to produce the octapeptide, angiotensin II, the biologically active molecule that plays an important role in the regulation of blood pressure and electrolyte balance. Although angiotensinogen mRNA has been identified in several tissues including the liver, kidney, brain, spinal cord, and aorta (1-3), most of the angiotensinogen present in plasma is derived from the liver (4).

Although factors affecting the hepatic synthesis of angiotensinogen have been studied

\footnotetext{
F Present address: Nissho Corporation, 3-9-3, Honjonishi, Kita-ku, Osaka 530, Japan.

* To whom correspondence should be addressed.
}

extensively (4-6), the mechanisms involved in the clearance of plasma angiotensinogen have never been elucidated. As documented previously in several studies, the level of plasma angiotensinogen increases dramatically after bilateral nephrectomy $(4,7)$. Although the mechanisms responsible is not completely understood, this increase is thought to be due to enhanced synthesis of angiotensinogen in the liver $(8,9)$. However, another possible mechanism in which the kidney carries out inactivation and/or excretion of angiotensinogen cannot be excluded. In fact, a recent report by Pedraza-Chaverri et al. have indicated that rat urine contains angiotensinogen (10), as measured by assay of angiotensin I liberated after incubation with porcine renin.

To understand the importance of urinary excretion of angiotensinogen in regulating the 
plasma level of this protein, we have studied urinary angiotensinogen in the rat using an anti-rat angiotensinogen antibody.

\section{MATERIALS AND METHODS}

\section{Treatment of animals and collection of urine}

Male F344 rats (Shizuoka Laboratory Animal Center, Hamamatsu, Japan), weighing $220-250 \mathrm{~g}$, were used. The rats were housed separately in metabolic cages at $22^{\circ} \mathrm{C}$, and 24 hr samples of urine were each collected into a bottle containing $10 \mu \mathrm{g}$ pepstatin and $2 \mathrm{mg}$ sodium azide in $1 \mathrm{ml}$ of distilled water to inhibit the action of renin. Each urine sample was centrifuged at $3,000 \times g$ for $15 \mathrm{~min}$ and the supernatant was obtained.

Acute inflammation was induced in rats by a single i.p. injection of lipopolysaccharide (LPS) (Staphylococcus typhosa 0910; Difco, Detroit, U.S.A.) at a dose of $0.5 \mathrm{mg} / \mathrm{kg}$. Daily 24-hr urine samples were collected for 4 days. Blood $(40 \mu 1)$ was also collected daily from the tail-tip of each animal into an EDTA-treated capillary tube by cutting with a razor. The tube was centrifuged at $1,000 \times g$ for $20 \mathrm{~min}$, and $15 \mu \mathrm{l}$ of plasma was obtained.

For the sodium depletion experiment, 8 animals were given furosemide $(5 \mathrm{mg}$, i.p.; Nacalai Tesque, Kyoto, Japan) and then fed a low-sodium diet containing $0.005 \%$ sodium (Oriental Yeast, Osaka, Japan) and distilled water for 21 days. Sodium-loading was accomplished by feeding 8 rats a high-sodium diet (1.6\%) for 21 days. Twenty-four-hour urine samples and plasma samples were collected on days 14 and 21 . For assay of the plasma renin concentration on day 21 , the rats were anesthetized with ether, and blood was collected from the abdominal aorta into a syringe containing a $1 / 10$ volume of $3.8 \%$ sodium citrate. Plasma was prepared by centrifugation at $1,000 \times g$ for $20 \mathrm{~min}$.

\section{Radioimmunoassay of angiotensinogen}

The angiotensinogen concentration in plasma or urine was measured by radioimmunoassay using $\left[{ }^{125} \mathrm{I}\right]$-labeled rat angiotensinogen and a rabbit antibody against rat angiotensinogen, as described previously (11). Assay of angiotensinogen in urine was done immediately after collecting the urine sample, since preservation of urine at low temperature caused the formation of insoluble precipitates containing angiotensinogen.

\section{Immunoaffinity chromatography of rat urine}

Anti-rat angiotensinogen immunoadsorbent was prepared by mixing $10 \mathrm{ml}$ of Affi-Gel 10 (Bio-Rad, Richmond, CA) in $0.1 \mathrm{M}$ sodium phosphate, $\mathrm{pH} 7.5$, with $90 \mathrm{mg}$ of an $\mathrm{IgG}$ preparation obtained from rabbit antiserum against rat angiotensinogen at $4^{\circ} \mathrm{C}$ for $4 \mathrm{hr}$. The gel was poured into a column $(0.9 \times 20$ $\mathrm{cm})$ and washed thoroughly with $0.2 \mathrm{M}$ glycine-HCl, pH 2.5, followed by $50 \mathrm{mM}$ Tris$\mathrm{HCl}, \mathrm{pH} 7.4$, containing $0.15 \mathrm{M} \mathrm{NaCl}, 0.1 \%$ Tween 20 and $0.02 \%$ sodium azide (equilibrium buffer). The urine sample was applied to the column and then washed with the equilibrium buffer. Adsorbed protein was eluted with $0.2 \mathrm{M}$ glycine- $\mathrm{HCl}, \mathrm{pH} 2.5$, and each fraction $(2.2 \mathrm{ml})$ was neutralized by adding $0.75 \mathrm{ml}$ of $1 \mathrm{M}$ Tris- $\mathrm{HCl}, \mathrm{pH}$ 8.0. Each fraction was subjected to the assay for angiotensinogen either by the direct method described above or by an indirect method measuring the amount of angiotensin I liberated by renin. In order to determine angiotensin I liberation, each fraction $(100 \mu \mathrm{l})$ was mixed with $25 \mu \mathrm{l}$ of $0.4 \mathrm{M}$ sodium phosphate, $\mathrm{pH} 6.5$, containing $10 \mathrm{mM}$ $\mathrm{Na}_{2}$ EDTA, $5 \mathrm{mM}$ o-phenanthroline and 0.5 $\mathrm{mM}$ captopril, and $25 \mu \mathrm{l}$ of partially purified rat renin $(2 \mu \mathrm{g})$, as described previously (12). The mixture was incubated for $90 \mathrm{~min}$ at $37^{\circ} \mathrm{C}$ followed by addition of $0.1 \mathrm{M}$ Tris- $\mathrm{HCl}, \mathrm{pH}$ 7.4 , containing $0.2 \%$ gelatin $(200 \mu \mathrm{l})$ and then heated in boiling water for $3 \mathrm{~min}$. Angiotensin I in the reaction mixture was measured by radioimmunoassay as described previously (12).

Immunoblot analysis of rat urinary angiotensinogen

Rat urine $(10 \mu \mathrm{l})$ and purified plasma angiotensinogen $(5 \mathrm{ng})$ were subjected to SDS- 
polyacrylamide gel electrophoresis in $10 \%$ gel according to the method of Laemmli (13). Purified plasma angiotensinogen was obtained from the plasma of nephrectomized rats as described previously (11). Western blotting was carried out by electrophoretic transfer to a nitrocellulose filter, and then the filter was soaked in a blocking solution containing $3 \%$ gelatin, $20 \mathrm{mM}$ Tris- $\mathrm{HCl}, \mathrm{pH} 7.5$, and $0.5 \mathrm{M}$ $\mathrm{NaCl}$ for $1 \mathrm{hr}$. The blot was then soaked in 200-fold-diluted anti-angiotensinogen serum for $2 \mathrm{hr}$ at room temperature, washed with the above buffer containing $0.05 \%$ Tween 20 , and incubated with horseradish peroxidase-labeled goat anti-rabbit IgG for $1 \mathrm{hr}$ at room temperaturc. Finally, the blot was washed with the above buffer containing $0.5 \%$ Tween 20 and processed for color development according to the instruction manual issued by Bio-Rad Laboratories.

\section{Miscellaneous methods}

Plasma renin concentration was assayed by measuring the amount of angiotensin I generated in plasma supplemented with an excess amount of renin substrate, as described previously (14).

\section{RESULTS}

Rat urine was found to contain an antigen that reacted with rabbit antibody against rat plasma angiotensinogen. Figure 1 shows a typical standard curve for the angiotensinogen radioimmunoassay, together with the displacements of $\left[{ }^{125} \mathrm{I}\right]$ angiotensinogen by a 24 -hr sample of rat urine. The displacement curve for urine was parallel to that of plasma angiotensinogen.

In order to characterize the angiotensinogen-like immunoreactivity in rat urine, the 24-hr urine was collected from 4 rats and applied directly to a column of anti-angiotensinogen immunoadsorbent (Fig. 2). Angiotensinogen-like immunoreactivity was adsorbed to the column and eluted with $0.2 \mathrm{M}$ glycine$\mathrm{HCl}, \mathrm{pH} 2.5$. When each fraction was incubated with an excess amount of rat renin, angiotensin I liberation was observed in fractions containing angiotensinogen-like immunoreac-

Rat urine ( $\mu 1 /$ tube)

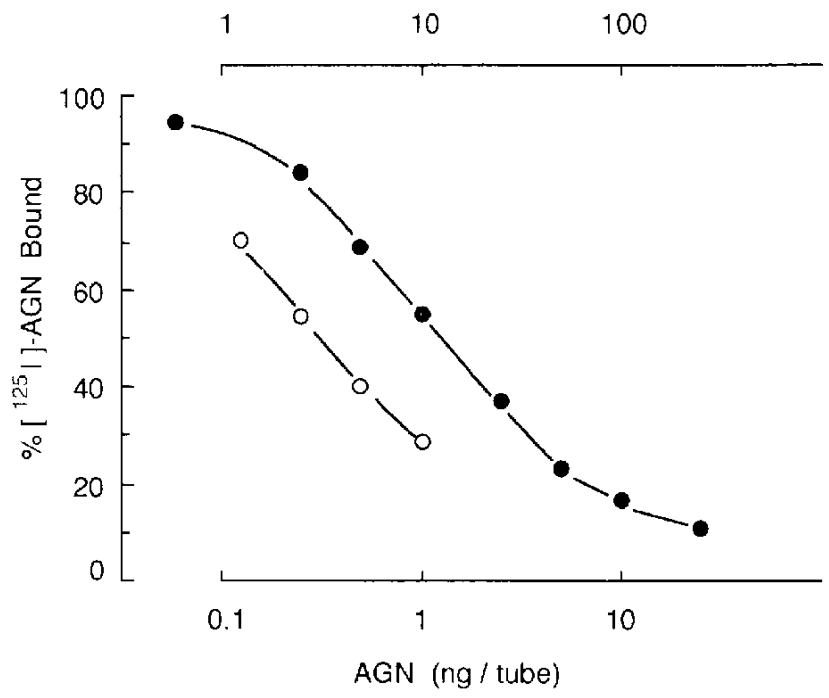

Fig. 1. Displacement of ${ }^{125}$ I-labeled angiotensinogen bound to antibody with rat plasma angiotensinogen and urine. Purifjed angiotensinogen from rat plasma $(0.0625-250 \mathrm{ng}, 0)$ and rat urine $(1.25-10 \mu 1, \bigcirc)$ were subjected to angiotensinogen radioimmunoassay. Each point represents the mean of duplicate assays. 


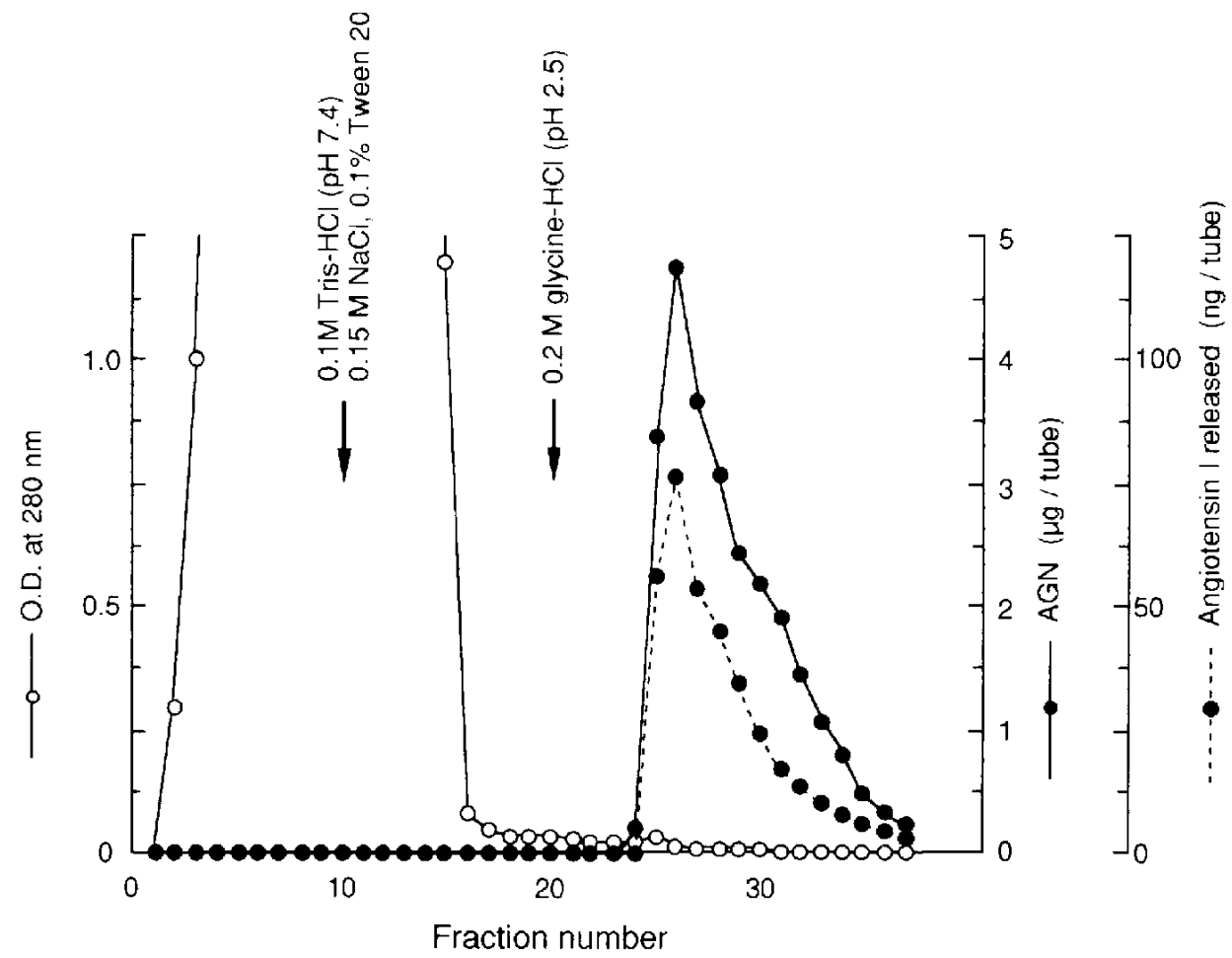

Fig. 2. Immunoaffinity chromatography of rat urinary angiotensinogen. Rat urine (36 ml) was applied to a column of anti-angiotensinogen IgG-agarose, previously equilibrated with $0.1 \mathrm{M}$ Tris- $\mathrm{HCl}, \mathrm{pH} 7.4$, in $0.15 \mathrm{M}$ $\mathrm{NaCl}$. The column was washed with the buffer containing $0.1 \%$ Tween 20 , followed by elution with $0.2 \mathrm{M}$ glycine- $\mathrm{HCl}, \mathrm{pH} 2.5$. The assay of angiotensinogen and generation of angiotensin I are described in the text.

tivity (Fig. 2). When the adsorbed fractions (fractions number 25 to 35 ) were pooled and assayed for immunoreactive angiotensinogen and angiotensin I liberation, the adsorbed fractions contained $25.5 \mu \mathrm{g}$ of immunoreactive angiotensinogen and generated $0.35 \mu \mathrm{g}$ of angiotensin I through the action of renin. Since the angiotensin I content of the intact form of rat angiotensinogen was expected to be 22.7 $\mu \mathrm{g} / \mathrm{mg}$ protein, $25.5 \mu \mathrm{g}$ of angiotensinogen in the adsorbed fractions would theoretically release $0.58 \mu \mathrm{g}$ of angiotensin 1, suggesting that $60 \%$ of the immunoreactive angiotensinogen in rat urine was the intact form of angiotensinogen, while the remainder was in the form of des-angiotensin I-angiotensinogen.

In order to estimate the molecular weight of the immunoreactive substance, $10 \mu \mathrm{l}$ of 24 -hr urine and $5 \mathrm{ng}$ of purified rat angiotensinogen were subjected to SDS-polyacrylamide gel electrophoresis followed by immunoblot analysis using anti-rat angiotensinogen antibody. As scen in Fig. 3, the immunoreactivity was localized in multiple bands between molecular weights of 50,000 and 60,000 , which corresponded to the immunoreactive bands of angiotensinogen purified from rat plasma. Multiple bands might result from differences in their carbohydrate content which could produce a slight change in molecular weight, as reported for plasma angiotensinogen (15). Thus the immunoreactive substance in rat urine was indistinguishable in its molecular weight from that of plasma angiotensinogen.

The amount of angiotensinogen excreted into rat urine was determined by radioimmunoassay to be $2.70 \pm 0.21 \mu \mathrm{g} /$ day $\quad(\mathrm{n}=8)$. Since several plasma proteins including albu- 

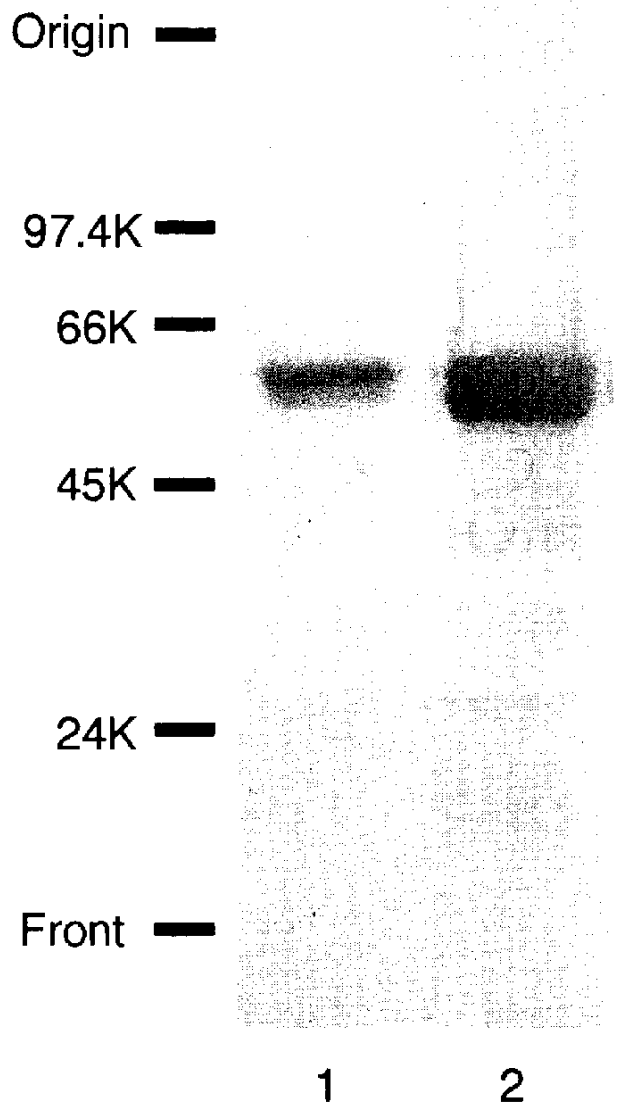

Fig. 3. Immunoblot analysis of immunoreactive angiotensinogen in rat urine. Purified plasma angiotensinogen (5ng, lane 1) and rat urine (10 $\mu \mathrm{l}$, lane 2) were subjected to SDS-polyacrylamide gel electrophoresis in a $10 \%$ gel, followed by Western blotting. Antigen to anti-angiotensinogen antibody was detected immunochemically as described in the text.

min and globulin are excreted into urine to some extent, the angiotensinogen found in urine appeared to be derived from plasma angiotensinogen. To verify this possibility, the urinary excretion of angiotensinogen was determined in rats after the injection of LPS, since acute inflammation is known to elevate the plasma concentration of angiotensinogen $(12,16)$ following increased synthesize of angiotensinogen in the liver (6). As shown in Fig. 4, the amount of angiotensinogen was increased approximately 7 -fold in 24-hr urine

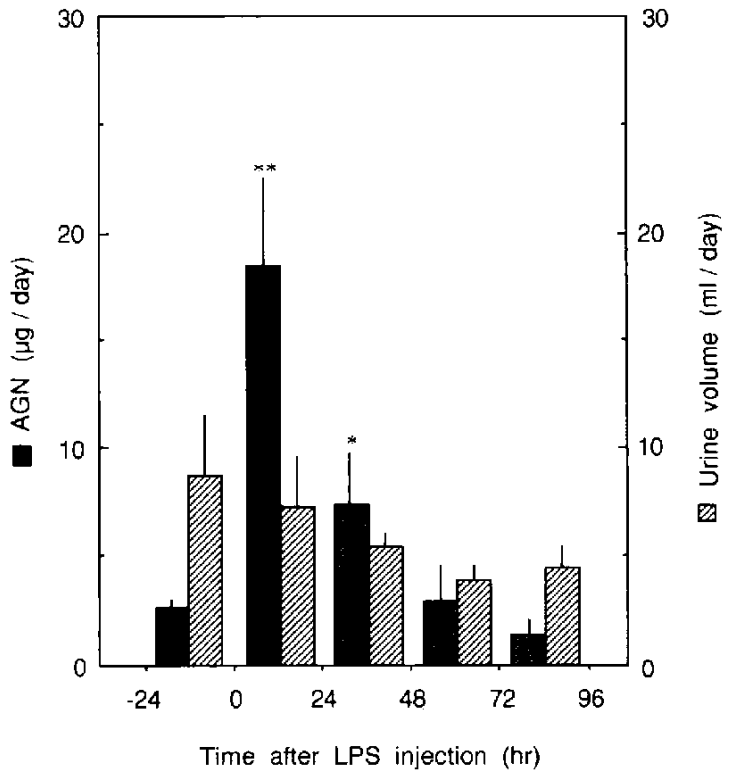

Fig. 4. Urinary excretion of angiotensinogen in rats following LPS injection. Rats were injected with LPS $(0.5 \mathrm{mg} / \mathrm{kg}$, i.p.) at $0 \mathrm{hr}$. Samples of 24 -hr urine were collected before and after LPS injection and subjected to angiotensinogen radioimmunoassay. Each bar represents the mean $\pm S$.D. for 8 rats. A single or double asterisk indicates significant difference from the level before LPS treatment ( ${ }^{*} \mathbf{P}<0.01$ and ${ }^{* *} \mathbf{P}<0.001$ ).

following LPS injection $(18.6 \pm 3.9 \mu \mathrm{g}$ compared with $2.7 \pm 0.21 \mu \mathrm{g}$ ). The plasma level of angiotensinogen was also increased from $72.3 \pm 10.8 \mu \mathrm{g} / \mathrm{ml}$ to $180.9 \pm 56.9 \mu \mathrm{g} / \mathrm{ml} 24$ hr after LPS injection (data not shown). The urinary level of angiotensinogen gradually fell to the normal level in 24-hr urine collected in the 48-72-hr period after LPS injection (Fig. 4). The plasma level of angiotensinogen was also reduced to $78.6 \pm 21.2 \mu \mathrm{g} / \mathrm{ml}$ after $48 \mathrm{hr}$.

Another possible origin of urinary angiotensinogen is the kidney, where angiotensinogen is synthesized (17) and may be secreted into the urine. This possibility was investigated by studying the urinary excretion of angiotensinogen in rats maintained on a low- or highsodium diet, since it has recently been demonstrated that the level of angiotensinogen mRNA in rat kidncy is increased on a lowsodium diet compared with a high-sodium one 


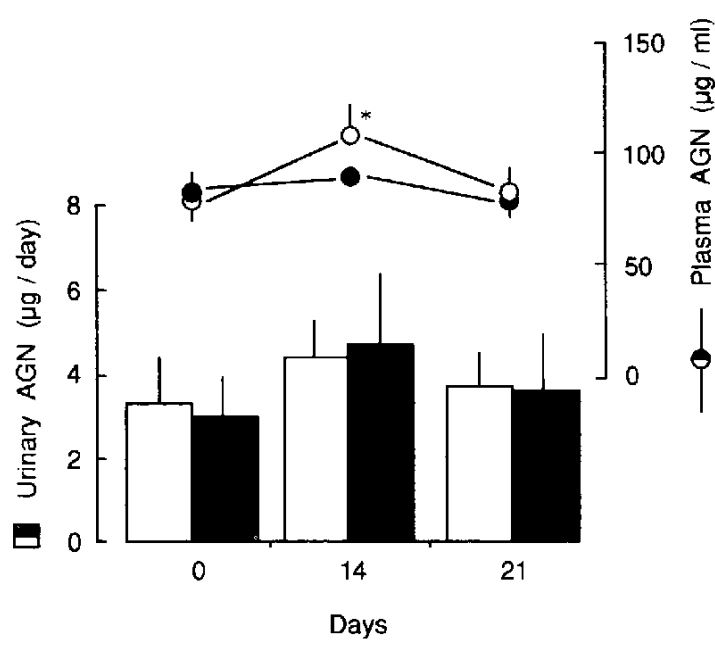

Fig. 5. Effects of a low- or high-sodium dict on urinary excretion or plasma level of angiotensinogen in rats. Rats were fed a low- or high-sodium diet for 21 days. Samples of 24-hr urine or plasma were collected on days 0,14 and 21 and subjected to angiotensinogen radioimmunoassay. Bars indicate the amount of angiotensinogen in the 24-hr urine of rats maintained on a low (clear bars) or high (solid bars) -sodium diet. Circles indicate the plasma level of angiotensinogen in rats maintained on a low (clear circles) or high (solid circles) -sodium diet. Values represent the mean \pm S.D. for 5 rats. An asterisk indicates a significant difference between groups on a low- and high-sodium $\operatorname{diet}\left({ }^{*} \mathbf{P}<0.05\right)$.

$(18,19)$. As shown in Fig. 5, there were no differences between sodium-depleted and sodium-loaded rats in the amounts of angiotensinogen excreted into 24-hr urine samples during the 21-day experimental period. The plasma level of angiotensinogen at 14 days was 1.2 times higher in rats maintained on the lowsodium diet than that on the high-sodium diet, but there was no difference at 21 days (Fig. 5). In contrast, plasma renin concentration was about 7 times higher on the low-sodium $\operatorname{diet}(283 \pm 53 \mathrm{ng}$ angiotensin $\mathrm{I} / \mathrm{ml} / \mathrm{hr})$ than on the high-sodium diet ( $42 \pm 4 \mathrm{ng}$ angiotensin $\mathrm{I} / \mathrm{ml} / \mathrm{hr}$ ), as reported previously (20).

\section{DISCUSSION}

In the present study, we demonstrated the presence of angiotensinogen in rat urine on the basis of the following evidence. First, rat urine contained a component that was crossreactive with antibody against rat angiotensinogen. The fact that the displacement curve of urine paralleled that of plasma angiotensinogen indicated that the antigen present in urine was immunologically identical to plasma angiotensinogen. Second, the immunoreactive angiotensinogen generated angiotensin I through the action of renin. Third, the molecular weights of the immunoreactive angiotensinogen in urine could not be distinguished from those of plasma angiotensinogen, as indicated by immunoblot analysis.

Recently, Pedraza-Chaverri et al, reported that both angiotensinogen and renin were excreted in rat urine (10). They measured the level of angiotensinogen by assay of angioten$\sin I$ after incubation of urine with an excess of porcine renin, and they reported that the urinary level of angiotensinogen in normal rats was $0.028 \mu \mathrm{g}$ angiotensin $\mathrm{I} /$ day. We measured urinary angiotensinogen by a direct radioimmunoassay, and the level in normal rats was determined to be $2.7 \mu \mathrm{g}$ angiotensinogen/day, which is equivalent to about $0.06 \mu \mathrm{g}$ angioten$\sin \mathrm{I} /$ day. Since about $60 \%$ of the urinary angiotensinogen was estimated as the intact form of angiotensinogen, our value for the amount of rat urinary angiotensinogen is close to that reported by Pedraza-Chaverri et al.

In order to determine the source of urinary angiotensinogen, we carried out two experiments: one involved the induction of acute inflammation, which causes elevation of plasma angiotensinogen following increased hepatic synthesis of angiotensinogen $(6,12,16)$, and the other examined the effect of a low- or high-sodium diet, which causes an increase or decrease of renal angiotensinogen mRNA, respectively $(18,19)$. It was found that LPS-induced acute inflammation caused elevation of plasma angiotensinogen with a concomitant increase in the urinary excretion of angiotensin- 
ogen. On the other hand, low/high dietary sodium did not influence the urinary excretion of this protein, whereas a significant clevation of plasma renin concentration was obscrved in rats maintained on a low-sodium compared with a high-sodium dict for 21 days, as reported previously $(20,21)$. In rats maintained for 14 days on a low-sodium diet, the plasma level of angiotensinogen was higher than that on a high-sodium diet. The mechanism is unknown, but a recent report showed that the angiotensinogen mRNA level in the liver was increased by a low-sodium diet (19). These rcsults indicate that urinary angiotensinogen is derived from plasma angiotensinogen, and not from that synthesized in the kidney.

Little and Sernia measured the production rate and metabolic clearance rate of angiotensinogen in the rat by constant-rate infusion and single injecction of ${ }^{125}$ I-labeled angiotensinogen (21). According to their results, the metabolic clcarance rate of angiotensinogen was $13 \mathrm{ml}$ plasma $/ \mathrm{hr} / \mathrm{kg}$, which corresponds to about $230 \mu \mathrm{g}$ angiotensinogen/hr per $250 \mathrm{~g}$ body weight, since the plasma level of angiotensinogen in the rat was about $70 \mu \mathrm{g} / \mathrm{ml}$. As urinary excretion of angiotensinogen was about $3 \mu \mathrm{g} / 24 \mathrm{hr}$ per animal, it is estimated that urinary excretion contributes less than $0.1 \%$ to the plasma clearance of angiotensinogen. Thus it is unlikely that the urinary excretion of angiotensinogen is a factor influencing the plasma level of this protein, except in some abnormal conditions, including nephrosis (10).

In conclusion, we have demonstrated the presence of angiotensinogen in rat urine, which is indistinguished from plasma angiotensinogen. The data indicate that urinary angiotensinogen is derived from plasma, but that the amount is too small to influence the plasma level of angiotensinogen.

\section{REFERENCES}

I Campbell, D.J., Bouhnik, J., Menard, J. and Corvol, P.: Identity of angiotensinogen precursors of rat brain and liver. Nature 308, 296-308 (1984)

2 Ohkubo, H., Nakayama, K., Tanaka, T. and Nakanishi, S.: Tissue distribution of rat angiotensinogen mRNA and structural analysis of heterogeneity. J. Biol. Chem. 261, 319-323 (1986)

3 Dzau, V.J., Ellison, K.E., Brody, T., Ingelfinger, J. and Pratt, R.E.: A comparative study of the distributions of renin and angiotensinogen messenger ribonucleic acids in rat and mouse tissues. Endocrinology 120, $2334-2338$ (1987)

4 Reid, I.A., Morris, B.J. and Ganong, W.F.: The renin-angiotensin system. Annu. Rev. Physiol. 40. $377-410$ (1978)

5 Chang, E. and Perlman, A.J.: Multiple hormones rcgulate angiotensinogen messenger ribonucleic acid levels in a rat hepatoma cell line. Endocrinology 121, $513-519(1987)$

6 Kageyama, R., Ohkubo, H. and Nakanishi, S.: Induction of rat liver angiotensinogen mRNA following acute inflammation. Biochem. Biophys. Res. Commun. 129, $826-832$ (1985)

7 Carretero, O. and Gross, F.: Renin substrate in plasma under various experimental conditions in the rat. Am. J. Physiol. 213, 695-700 (1967)

8 Campbell, D.J. and Habener, J.F.: Angiotensinogen gene is expressed and differentially regulated in multiple tissues of the rat. J. Clin. Invest. 78 , $31-39$ (1986)

9 Iwao, H., Kimura, S. Fukui, K., Nakamura, A., Tamaki, T., Ohkubo, H., Nakanishi, S. and Abe, $\mathrm{Y}$ : Elevated angiotensinogen mRNA levels in rat liver by nephrectomy. Am. J. Physiol. 258, E413E417 (1990)

10) Pedraza-Chaverri, J., Cruz, C., Ibarra-Rubio, M.E., Hernandez, C., Tapia, E. and Pena, J.C.: Urinary excretion of renin and angiotensinogen in nephrotic rats. Nephron 57, 106-108 (1990)

11 Itoh, N., Matsuda, T., Ohtani, R. and Okamoto, $H$.: Angiotensinogen production by rat hepatoma cells is stimulated by $B$ cell stimulatory factor 2/interleukin-6. FEBS Lett. 244, 6-10 (1989)

12 Okamoto, H., Hatta, A., Itoh, N., Ohashi, Y. Arakawa, K. and Nakanishi, S.: Acute-phase responses of plasma angiotensinogen and T-kininogen in rats. Biochem. Pharmacol. 36, $3069-3073$ (1987)

13 Laemmli, U.K.: Clcavage of structural proteins during the assembly of the head of bacteriophage T4. Nature 227, 680-685(1970)

14 Ohtani, R., Ohashi, Y., Muranaga, K., Itoh, N 
and Okamoto, H.: Changes in activity of the renin-angiotensin system of the rat by induction of acute inflammation. Life Sci. 44, 237-241 (1989)

15 Bouhnik, J., Clauser, E., Strosberg, D., Frenoy, J.-P., Menard, J and Corvol, P.: Rat angiotensinogen and des(angiotensin I) angiotensinogen: Purification, characterization, and partial sequencing. Biochemistry 20,7010-7015 (1981)

16 Okamoto, H., Ohashi, Y. and Itoh, N.: Involvement of leukocytc and glucocorticoid in the acutephase response of angiotensinogen. Biochem. Biophys. Res. Commun. 145, 1225 - 1230 (1987)

17 Dzau, V.J. and Ingelfinger, J.R.: Molecular biology and pathophysiology of the intrarenal renin-angiotensin system. J. Hypertens. 7, S3-S8 (1989)

18 Ingelfinger, J.R., Pratt, R.E., Ellison, K. and
Dzau, V.J.: Sodium regulation of angiotensinogen mRNA expression in rat kidney cortex and medulla. J. Clin. Invest. 78, $1311-1315$ (1986)

19 Iwao, H., Fukui, K., Kim, S., Nakayama, K. Ohkubo, H., Nakanishi, S. and Abe, Y.: Sodium balance effects on renin, angiotensinogen, and atrial natriuretic polypeptide mRNA levels. Am. J. Physiol. 255, E129-E136 (1988)

20 Herrmann, H.C and Dzau, V.J.: The feedback regulation of angiotensinogen production by components of the renin-angiotensin system. Circ. Res. 52, $328-334$ (1983)

21 Little, M.H. and Sernia, C.: Production and metabolic clearance of angiotensinogen in conscious rats as measured by steady-state isotope dilution. J. Endocrinol. 112, $391-397$ (1987) 